\title{
Antireflective moth-eye structures on curved surfaces fabricated by nanoimprint lithography (Erratum)
}

\section{Michael Haslinger, Amiya Moharana, Michael Mühlberger}

Michael J. Haslinger, Amiya R. Moharana, Michael Mühlberger, "Antireflective moth-eye structures on curved surfaces fabricated by nanoimprint lithography (Erratum)," Proc. SPIE 11177, 35th European Mask and Lithography Conference (EMLC 2019), 1117716 (24 February 2020); doi: $10.1117 / 12.2569999$ SPIE. Event: 35th European Mask and Lithography Conference, 2019, Dresden, 


\section{Antireflective moth-eye structures on curved surfaces fabricated by nanoimprint lithography (Erratum)}

Michael J. Haslinger, Amiya R. Moharana, and Michael Mühlberger

Profactor $\mathrm{GmbH}$ (Austria)

Proceedings Volume 11177, 35th European Mask and Lithography Conference (EMLC 2019); $111770 \mathrm{~K}$ (2019) https://doi.org/10.1117/12.2535683

Event: 35th European Mask and Lithography Conference (EMLC 2019); Dresden, Germany

Online Publication Date: 29 August 2019

Erratum Published: 24 February 2020

A revised version of this manuscript was published on 24 February 2020. The article contained different pictures in low resolution. The picture in Figure $3 \mathrm{c}$ ) and Figure 4 a) have been replaced by more highquality images. 